\title{
Implementation of Channel Estimation and Modulation Technique for MIMO System
}

\author{
Mrs. VEENA M.B. ${ }^{1}$ \& Dr.M.N.SHANMUKHA SWAMY ${ }^{2}$ \\ Research scholar $^{1}$, Professor $^{2}$ \\ ECE department, SJCE, Mysore. \\ Karnataka, India \\ e_mail: veenahod@rediffmail.com ${ }^{1}$, mnsjce@yahoo.co.in ${ }^{2}$
}

\begin{abstract}
Future wireless communication system have to be designed to integrate features such as high data rates, high quality of service and multimedia in the existing communication framework. Increased demand in wireless communication system has led to demand for higher network capacity and performance. Higher bandwidth, optimized modulation offer practically limited potential to increase the spectral efficiency. Hence MIMO systems utilizes space multiplex by using array of antenna's for enhancing the efficiency at particular utilized bandwidth. MIMO use multiple inputs multiple outputs from single channel. These systems defined by spectral diversity and spatial multiplexing. The aim of this paper is to design and implement of channel estimation method and modulation technique for MIMO system. The design specifications are obtained using MATLAB. The RTL coding is carried for the design to be implemented on Xilinx FPGA.
\end{abstract}

Key words: MIMO, FPGA, Space multiplex, RTL.

\section{Introduction}

MIMO describes the ways to send data from multiple users on the same frequency/time channel using multiple antennas at the transmitter and receiver end. A transmitter/receiver system uses multiple antennas not only for transmitting data between corresponding antennas but also between adjacent antennas. The data is received in the form of MIMO Channel Matrix. A top level MIMO system is shown in Fig.1 MIMO system is used in many applications like WiMax, WiFi, WLANs, and many more signal processing applications.

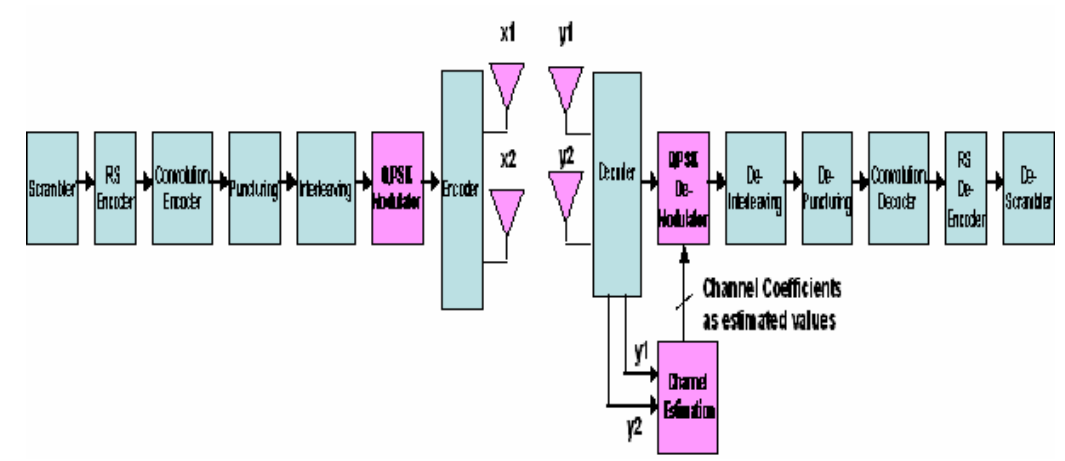

Figure 1 : MIMO System [1]

Blocks in MIMO system are scrambler/descrambler, RS encoder/decoder, convolution encoder/decoder, puncturing/de-puncturing, interleaver/de-interleaver, modulator/ demodulator, diversity or space time encoder/decoder and channel estimation. This paper aims at design and

DOI : 10.5121/ijwmn.2011.3211 
implementation of a simple and most efficient channel estimation method and a good modulation technique for increasing the channel capacity, bandwidth, increasing bit rates and eliminates intersymbol interference.There are well-known training based channel estimation methods are; least square (LS), Minimum Mean Square Estimation (MMSE), Least mean square (LMS), Recursive Least Square (RLS). In this paper the main focus is on simple LS algorithm, which is simple to analyse and efficient. The comparison of LS is not performed. The main aim is to reduce the computational complexity of channel estimation using LS algorithm and implementing 2x2 MIMO system using QPSK modulation technique.

\section{Literature Survey}

Wout Joseph, et.al. [1] proposed an algorithm for the performance study on IEEE 802.16-2004 for different conditions like for different channel models and MIMO system. Chia-Liang Liu [4] explained the impact of I/Q imbalance on QPSK-OFDM-QAM Detection. The in-phase and the quadrature phase components are very important component in QPSK. Yantao Qiao, et al.[5], has made a research on an iterative algorithm of least square channel estimation in MIMO OFDM systems. The main objective of this paper is an iterative channel estimation algorithm for MIMO OFDM is proposed. Sarod Yatawatta, et.al.[7] proposed a solution for minimizing the energy spent on during the channel estimation when subjected to known error and delay when timing symbols are transmitted. The minimization of energy is carried by reducing the hardware, also by using a low rank equalization at the receiver. Benoit Le Saux, et.al, [8] proposed a MIMO system with OFDM has greater potential like reduction in inter-symbol interference, decrease in fading, increase in bandwidth, and increase in data rates. The performance of MIMO system degrades due to inaccurate channel estimation over frequency selective fast-varying channels.

Riza Abdolee, et.al.[10] proposed a method to reduce the computational complexity of channel estimation algorithm for MIMO-OFDM. Channel estimation is high intensive which suffer from high computational complexity. Solution for high efficient channel estimation and simplified computational complexity is stated. Deseada Bellido, et.al, [11] proposed LS channel estimation algorithm for MIMO-OFDM. This evaluation has been made using pilot design rules that guarantee a bounded error level for the estimation. This method is used for estimation of the channel matrix. Markus Myllyla, et.al, [12] proposed a method for performance evaluation of 2 FPGA implementation of a LMMSE based detector for radio channels.

\section{Channel Estimation and QPSK Design}

In this 2x2 MIMO system is designed. MIMO system [3] has multiple transmitter antennas and multiple receiver antennas so that the data is transmitted in parallel.. The MIMO system is designed with least square channel estimation method and QPSK modulation technique. Each module in MIMO system is explained in detail.

2x2 MIMO system is shown below in Fig.2 MIMO system has following blocks.

1) Least Square (LS) channel estimation block

2) QPSK modulator/demodulator

3) $2 \times 2$ Ideal MIMO Channel ( 2 transmitter $\& 2$ Receiver antennas). 


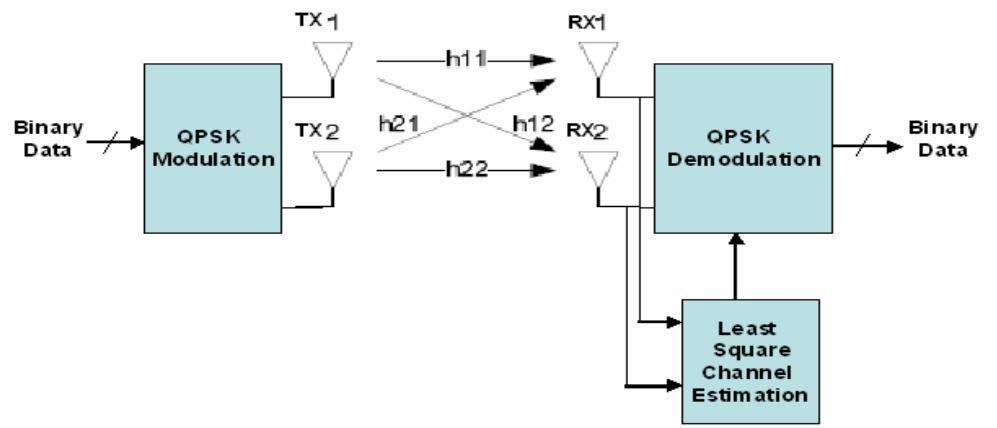

Figure $2: 2 \times 2$ MIMO system

\subsection{Least Square Channel Estimation Method}

In communication systems, channels are usually multi-path channels, which cause inter-symbol interference in the received signal. Channel estimators[5] require the channel impulse response (CIR). The channel estimation is based on the known sequence of bits called training sequence which is unique for each transmitter. Here the known training sequence is transmitted so that the channel coefficients are obtained. There are different standards used for transmitting training sequence like IEEE 802.16 standard.

\subsubsection{Channel estimator for single transmitter and single receiver}

In any communication system noise get added in the channel with the signal transmitted. The digital signal transmitted over a faded multi-path channel ' $\boldsymbol{h}$ '. The noise get added which is modeled as additive white Gaussian noise ' $\boldsymbol{n}$ ' as shown in Fig. 3 The demodulation problem is to detect the transmitted bits ' $x$ ' from the received signal ' $y$ '. The received signal also has channel coefficients multiplied. The detector needs these channel estimates for that specific channel and channel estimation device. The system is shown by the equation (3.1).

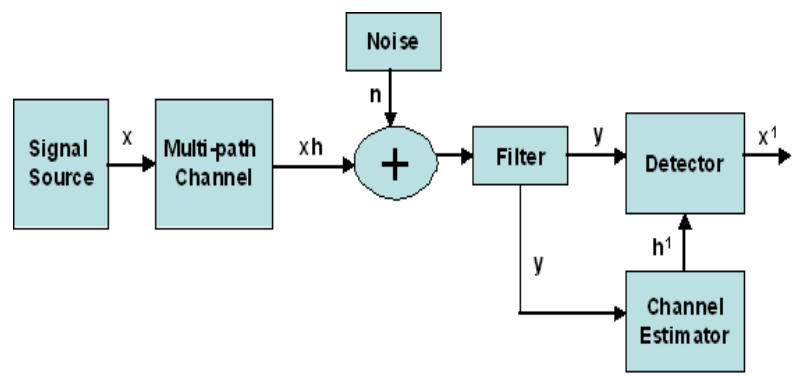

Figure 3 : Block diagram of a noise corrupted system

The received signal $y$ is given as

$y=M h+n$

$h=\left[h_{0} h_{1} \ldots . . . . h_{L}\right]^{T}$

In each transmission burst the transmitters sends a unique training sequence which has elements $\mathrm{m}_{\mathrm{i}} \in\{-1,+1\}$.Here $M$ is the matrix for training sequence. Hence the final least square channel estimation equation is given by equation (3.3).

$h_{L S}^{l}=\left(M^{H} M\right)^{-1} M^{H} y$

\subsubsection{Channel estimator for $2 \times 2$ channels}

$2 \times 2$ joint channel estimation is considered for the MIMO design. The channel is considered to be ideal wherein noise is not taken in account. The sequence ' $x$ ' is taken from 2 different 
transmitters and 4 channels are considered as shown in Fig.4 From each transmitter unique training sequences are transmitted in concatenation with the QPSK modulated data. The training sequence is transmitted for the identification of the transmitter from which the data is obtained at the receiver end. Here diversity technique is used but the alamouti encoder and decoder is not used.

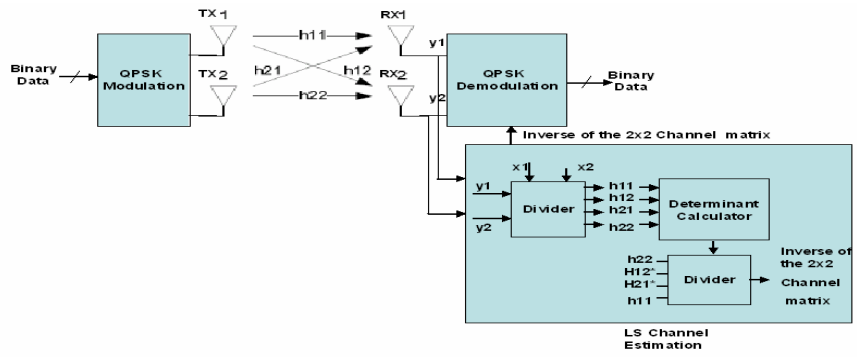

Figure 4 : Block showing QPSK modulator/demodulator with LS channel estimation

The $2 \times 2$ channel estimation block diagram is shown in Fig.2. Initially the training sequence is transmitted so that the channel coefficients are calculated. First the transmitter is made active and the training sequence is sent. The tx 2 is made inactive i.e. nothing is sent from tx 2 . The $h 11$ and $\mathrm{h} 12$ channel coefficients are obtained. Then the tx 1 is made inactive and tr 2 is sent through tx2. Then the $h 21$ and $h 22$ coefficients obtained by simplifying the equations (3.3). The data obtained at the receivers $\mathrm{rx} 1$ and $\mathrm{rx} 2$ are shown by the equations (3.4.and 3.5).

$$
\begin{aligned}
& \mathrm{y} 1=\mathrm{x} 1 * \mathrm{~h} 11+\mathrm{x} 2 * \mathrm{~h} 21 \\
& \mathrm{y} 2=\mathrm{x} 1 * \mathrm{~h} 12+\mathrm{x} 2 * \mathrm{~h} 22
\end{aligned}
$$

After which the transmitted bits are decoded by multiplying $y$ with the inverse of the $2 \times 2$ channel matrix $h$.

$$
\mathrm{h}^{1}=\mathrm{y} / \mathrm{x}
$$

\subsection{QPSK modulator/ demodulator}

PSK is a digital modulation technique which is most commonly used modulation technique in present digital communication systems. In PSK modulation, the phase of the carrier is altered in accordance with the input binary coded information. The PSK is further subdivided into BPSK, 8-PSK, 16-PSK, QPSK, DPSK. In binary phase shifting keying the transmitted signal is sinusoid of fixed amplitude, has fixed phase.

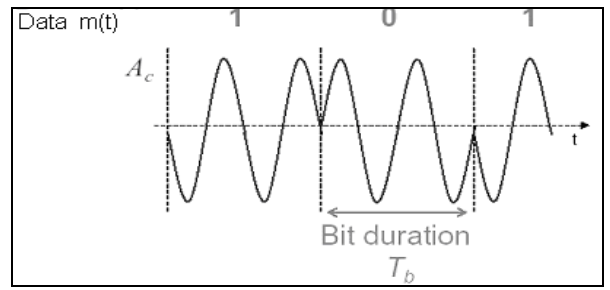

Figure 5 : Phase Shift Keying [1]

QPSK [4] is a phase modulation scheme, used in constellation mapping. Here the input bits stream is converted into complex stream using equation (3.7) and where the I and Q both are in phase with I-out and Q-out respectively. QPSK modulator accepts the binary bits as inputs taken as a symbol and converts them into complex value. QPSK takes only 4 symbols and generate its complex value in this fashion.

$\mathrm{D}=(\mathrm{I}+\mathrm{jQ}) * \mathrm{~K}_{\mathrm{MOD}}$ 
Where $\mathrm{K}_{\mathrm{MOD}}=1 / 1.414$

Table 1 : Table showing the Inputs and Outputs of QPSK Modulator

\begin{tabular}{|c|c|c|}
\hline $\begin{array}{c}\text { Input } \\
\text { Bits }\end{array}$ & I-out & Q-out \\
\hline 00 & -1 & -1 \\
01 & -1 & +1 \\
10 & +1 & -1 \\
11 & +1 & +1 \\
\hline
\end{tabular}

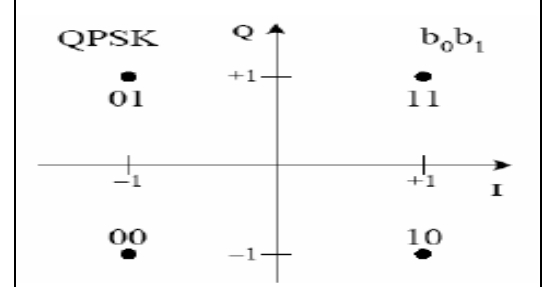

Figure 6 : QPSK Constellation Mapping

\subsection{Working of 2x2 MIMO System}

The 2x2 MIMO system has different blocks like QPSK modulator, ideal channel, channel estimation block and QPSK demodulation. QPSK modulator takes the binary bits as input. Here the inputs bits are taken as symbols. For example 00, 01, 10,11 are 4 inputs symbols for QPSK. The symbols are converted into complex values as shown in table 1 . The complex output of the modulator is appended with the training sequence at 2 transmitters. Here the diversity technique is used to send the same data (QPSK modulated output) with different training sequences. The channel coefficients are multiplied with the modulated data. Here 4 paths are considered or $2 \times 2$ channels as shown in Fig.7

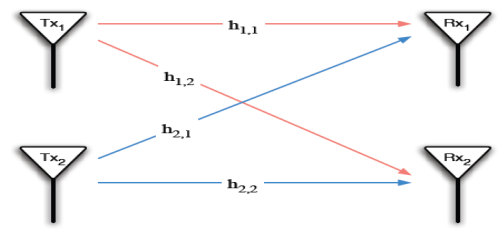

Figure 7 : Communication Channel for 2x2 MIMO System [13]

The channel is an ideal channel i.e. the noise is not considered. The reliability is increased by employing the diversity technique i.e. transmitting the same information across multiple channels. If one of the channels is not used or if the data is lost in space then the information/data can still be recovered from redundant transmission over the channels and hence the reliability of the communication system is improved.

The data transmitted from transmitter tx1 across the channels hand received by both the receiver's rx1 and rx2. Here the use of the available channels is done to increase the capacity and reliability. Then the received sequence is taken into the channel estimation block to find the estimated channel coefficients. The received data from LS channel estimator [5] block are the given to the decision block in the QPSK demodulator block to get back the sent binary sequence. The transmitted sequence is the checked with the received sequence at the QPSK modulator and the Demodulator block respectively. 


\section{Simulation \& Implementation Overview}

The MATLAB specifications of MIMO system from MATLAB are shown below.

1) $2 \times 2$ MIMO Technique: 2 transmitters and 2 Receiver antennas

2) QPSK modulation technique

3) Number of bits transmitted 64bits

4) Number of iterations $=100$

5) Signal to noise ratio $\mathrm{SNR}=[0: 3: 9]$ i.e. $0,3,6,9$ in $\mathrm{dB}$

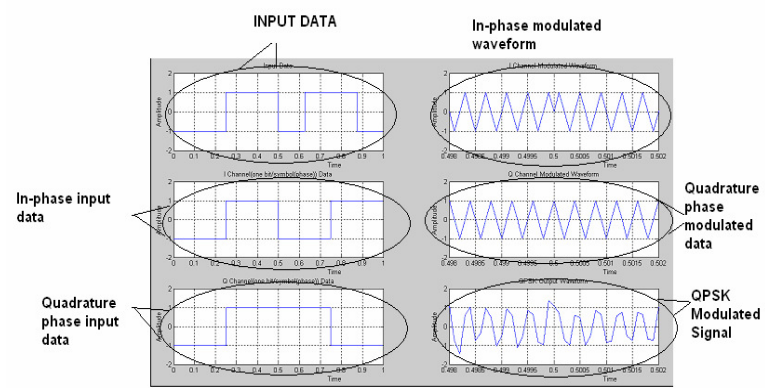

Figure 8 : QPSK modulator MATLAB outputs

QPSK modulated output is shown in Fig.8 Input binary data is sent through QPSK modulator, the data taken into is in the form of symbols. The in-phase and quadrature waveform and the QPSK modulated data are shown in Fig.8 The modulated data sent through the noisy channel as shown in Fig.4 the data get mixed with the channel coefficients and the noise. The bit error rate with respect to signal to noise

ratio the error rate goes on reducing as shown in Fig.9 (a). The error rate goes on reducing from $10^{0}$ to $10^{-1}$ as the SNR is from 0 to $10 \mathrm{~dB}$. As the number of iterations increases the error at the output which is given as the difference between the input and the output signal becomes as zero. The error plot is shown in Fig.9 (b) is for 100 iterations. There is a mismatch at 2 points and the difference is plotted. These plots as shown in Fig.9 are for LS channel estimation method.

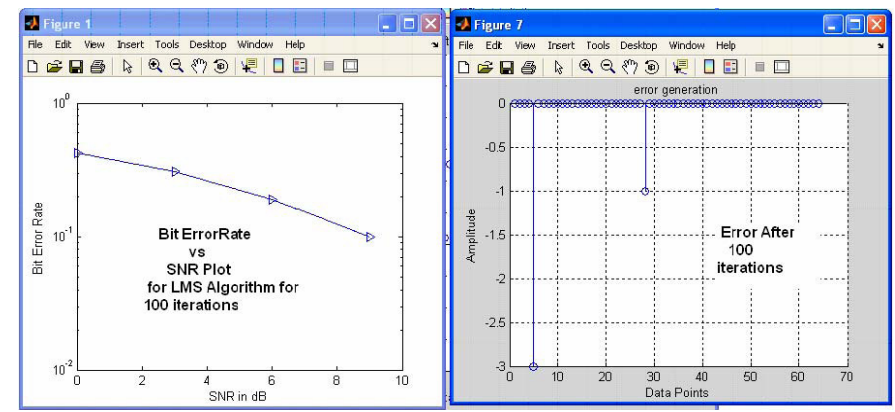

(a)

(b)

Figure 9 : Graph showing BER vs SNR plot and Error generation after 100 iterations

As the number of iterations increase the error reduces so that the BER vs SNR ratio goes on reducing and becomes negligible. Whenever there is a mismatch between the input and the output sequence of MIMO system then the difference is shown as the error.

The plots shown in Fig.10 are the input to a system is a random data generated using 'randn' in MATLAB. The random data generated is fed to the QPSK modulated. The data get modulated and then the modulated output is sent through the QPSK demodulator. The demodulated output 
same as input as shown in Fig.10 The plots as shown in Fig.10 are from MATLAB. Here the input is taken as 64 symbols.

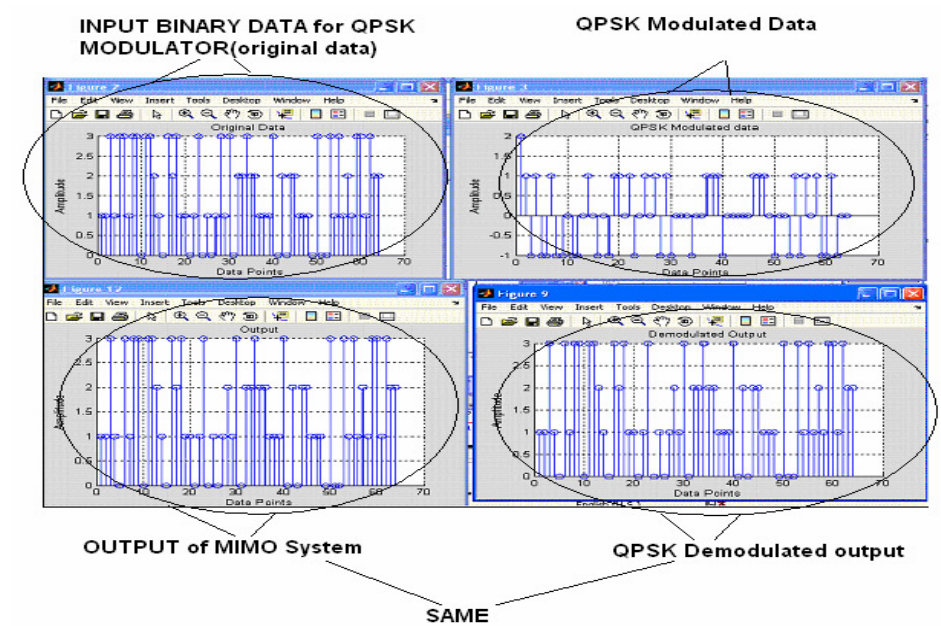

Figure 10 : Plots showing the input modulated output and demodulated output

$2 \times 2$ MIMO system is designed using Verilog-HDL and simulated in ModelSim. Each block is separately verified for its functionality. Each block is synthesized and the integrated output is also verified by implementing Xilinx FPGA. The top block of 2x2 MIMO system block diagram from Xilinx is shown in Fig.11 All the blocks in 2x2 MIMO system like QPSK modulator/demodulator, ideal channel and the LS channel estimation blocks are integrated and the connections are shown in Fig.11 with inputs and the outputs.

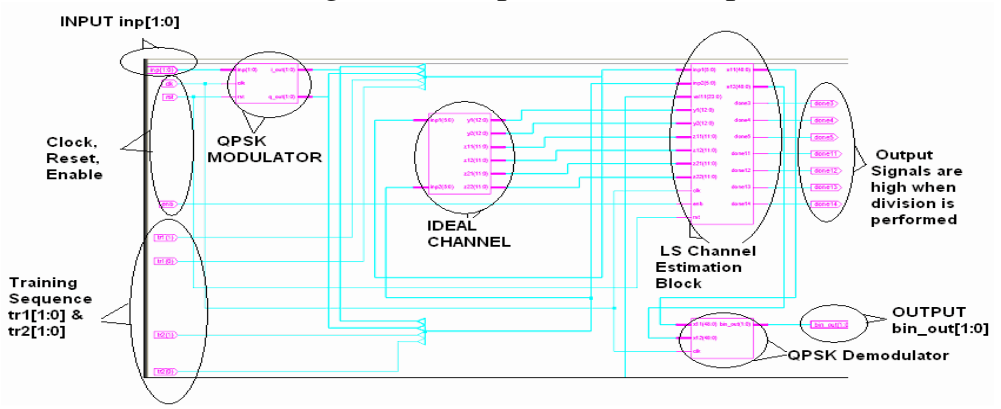

Figure $11: 2 \times 2$ MIMO System in Xilinx

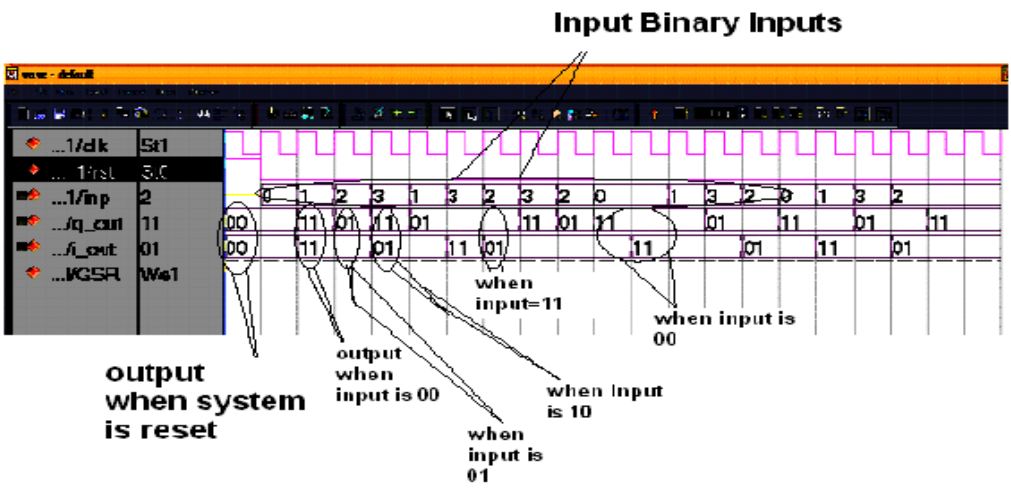

Figure 12: Waveform showing QPSK modulated output from ModelSim 
The waveform shown in Fig.12 shows the QPSK modulated output. As explained before each symbol is displayed as complex value. Similarly symbol 00 gives in phase value as 11 and quadrature phase value as 11 as shown in Table 2 .

Table 2: Table showing Binary Symbol representation as i_out and q_out

\begin{tabular}{|c|c|c|}
\hline Binary Symbol & I_out & Q_out \\
\hline 00 & 11 & 11 \\
\hline 01 & 11 & 01 \\
\hline 10 & 01 & 11 \\
\hline 11 & 01 & $\mathbf{0 1}$ \\
\hline
\end{tabular}

The Fig.13 shows the waveform for ideal channel. This block is a completely combinational block. Here the data from QPSK is passed so that the channel coefficients are multiplied with it. Here as explained before the training sequence transmitted from tr 1 is $01\{+1\}$ and from transmitter 2 is $11\{-1\}$.

Table 3: Table Displaying the Demodulated Output

\begin{tabular}{|c|c|c|}
\hline $\mathbf{x 1 1}$ & $\mathbf{X 1 2}$ & $\begin{array}{c}\text { Output of QPSK } \\
\text { demodulator }\end{array}$ \\
\hline 65 & 67 & $00=>0$ \\
\hline 56 & 58 & $01=>1$ \\
\hline 31 & 33 & $10=>2$ \\
\hline 22 & 24 & $11=>3$ \\
\hline
\end{tabular}

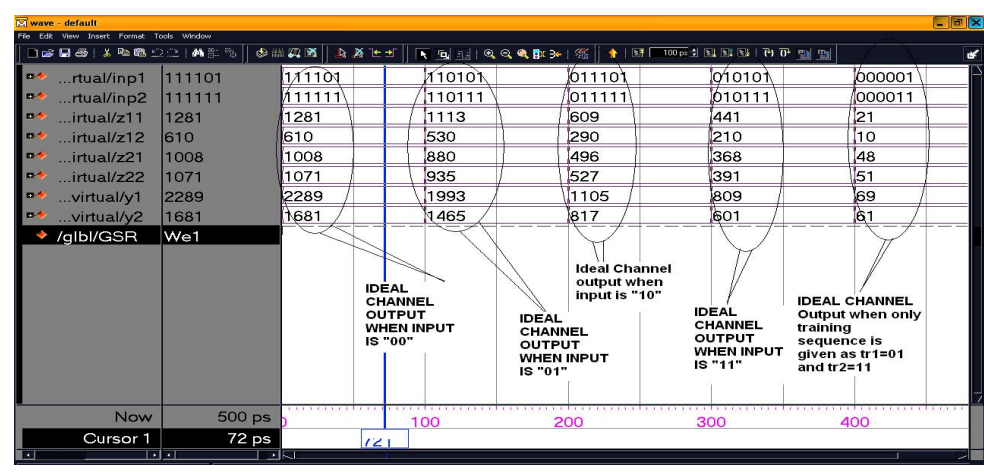

Figure 13: Waveform showing ideal channel outputs

The channel coefficients are taken from the MATLAB and are multiplied by $2^{\wedge} 10$ i.e. the fixed point conversion is done for the channel coefficients. The inp1 is multiplied with the h11, h12 and inp2 is multiplied with the $\mathrm{h} 21$ and $\mathrm{h} 22$. Then the $\mathrm{z} 11$ is added with $\mathrm{z} 21$ and $\mathrm{z} 12$ added with $\mathrm{z} 22$ is to get $\mathrm{y} 1$ and $\mathrm{y} 2$ respectively. The $\mathrm{y} 1$ and $\mathrm{y} 2$ are received at the receiver. For different input sequences the waveform is shown in Fig.13 


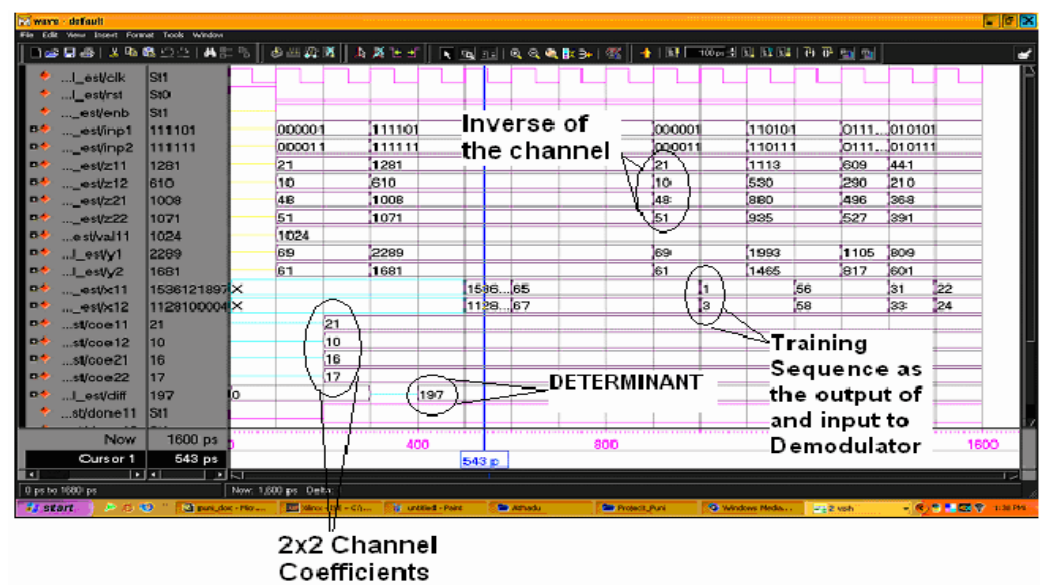

Figure 14: Waveform showing the LS channel Estimation block outputs

The outputs of the ideal channel are fed as inputs to the LS channel estimation where the inverse of channel coefficients is calculated. The channel is $2 \times 2$ and the matrix is $2 \times 2$ square matrix. Then the inverse of the channel matrix is multiplied with the received sequence. As shown in equation (2.6). The Fig.14 shows the LS channel estimation outputs wherein the inverse of the square matrix is calculated. The determinant is calculated for inverse matrix calculation.

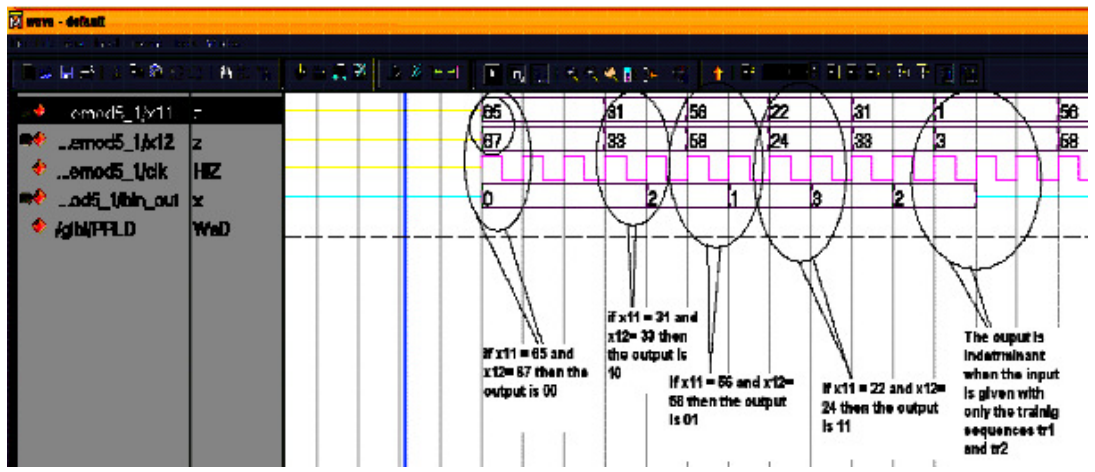

Figure 15 : Waveform showing QPSK demodulated output

The outputs of the LS channel estimation block are fed to the decision device used in QPSK demodulator which defines the value $\mathrm{x} 11$ and $\mathrm{x} 12$ as the values. The values in the required range i.e. the value $\mathrm{x} 11$ and $\mathrm{x} 12$ are 31 and 33 then the output should be '10' i.e. 2 as shown in Fig.15 Similarly, for different values of $\mathrm{x} 11$ and $\mathrm{x} 12$ the outputs of QPSK demodulator are shown in Table 3.

The integrated 2x2 MIMO system design output is as shown in Fig.16 the waveform shows the $2 \times 2$ MIMO system output. Here the clock is applied and the reset is made high then the system is made reset. Then the reset signal is made low and the enable signal is made high. The inp[1:0] is the given the value as the required symbol to transmit. The training sequence $\operatorname{tr} 1$ is given as +1 and training sequence $\operatorname{tr} 2$ is given as -1 . Then the output bin_out[1:0] is obtained as the input inp[1:0] 


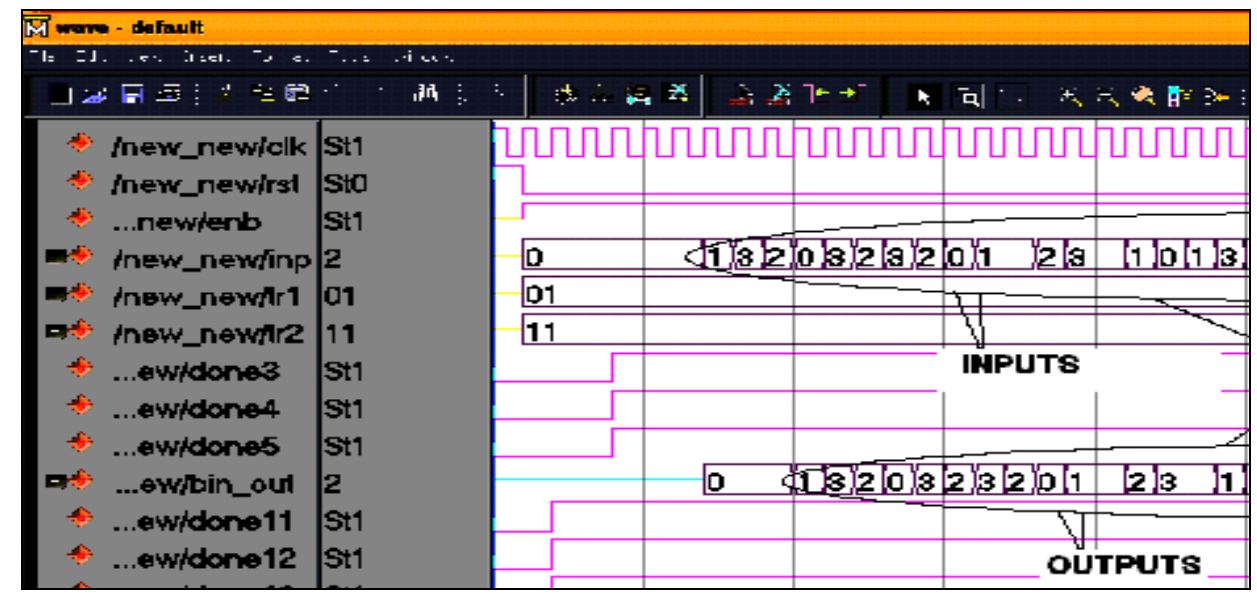

Figure 16. 2x2 MIMO System output waveform in ModelSim

\section{Conclusion}

The MIMO system design is simulated in MATLAB to arrive at the specifications. The RTL code is successfully simulated in ModelSim. The design synthesized and implemented on Virtex2Pro FPGA board and the results were validated using Logic Analyzer. The synthesis and timing is verified and the timing is met for both setup and hold in DC and PT. DFT is also carried without timing violation. The operating frequency of the design is $13 \mathrm{MHz}$ obtained from Xilinx. The top design takes about 3999 number of slices out of 4928 slices i.e. in Virtex2Pro the device selected is $2 \mathrm{vp} 7 \mathrm{ff} 896$ at speed grade of 6 with operating frequency $7.27 \mathrm{MHz}$. The $2 \mathrm{vp} 30 \mathrm{ff} 896$ is selected with a speed grade of 6 , the slices are 1902 out of 13696 i.e. $13 \%$ resources usage and operating frequency is $13.388 \mathrm{MHz}$ and minimum period of $74.691 \mathrm{~ns}$. Timing verified is all met with positive slack with zero violations.

\section{Acknowledgement}

Authors would like to thank the Management Vemana Institute of Technology, KRJS, Bangalore, for funding and providing necessary help to complete this paper.

\section{References}

[1] Joseph, W. Reynders, W. Debruyne, J. and Martens, L., "Influence of Channel Models and MIMO on the Performance of a System based on IEEE 802.16", Wireless Communications and Networking Conference, ISBN 1-4244-0659-5, pp.1826-1830, 11-15 March, 2007.

[2] Simon S Haykin and Michael Moher, "Modern Wireless Communication", Second Edition, Prentice Hall publication 2004, ISBN-13: 9780130224729.

[3] George Tsoulos, “MIMO System Technology For Wireless Communications", Revised Edition, CRC Publisher, 2006.

[4] Chia-Liang Lui, "Impacts of I/Q Imbalance on QPSK -OFDM-QAM Detection", IEEE Transactions on Consumer Electronics, vol. 44, no. 3, pp. (984-989), August, 1998.

[5] Yantao Qiao, Songyu $\mathrm{Yu}$, Pengcheng $\mathrm{Su}$ and Lijun Zhang, "Research on an Iterative Algorithm of LS Channel Estimation in MIMO OFDM Systems", IEEE Transactions on Broadcasting, vol. 51, no. 1, pp. (149-153), March 2005.

[6] S.Cui, A.J.Goldsmith and A.Bahai, "Energy Efficiency in MIMO and cooperative MIMO techniques in Sensor Networks", IEEE Journal on Selective Areas in Communication, vol. 22, no. 6, pp. (1089-1098), August 2004. 
[7] Yatawatta,S, Petropulu,A.P and Graff,C.J, "Energy efficient Channel Estimation in MIMO Systems",IEEE international conference on Acoustics,Speech and Signal processing, vol.4, no.1, pp.(317-320), 18-23 March,2005.

[8] Saux,B.L and Helard,M., "Iterative Channel Estimation based on Linear Regression for a MIMO-OFDM System",IEEE interanational Conf. on Networking and Communications,vol.1, no.1, pp.( 356-361), 19-21June, 2006.

[9] Changchuan Yin, Jingyu Li, Xiaolin Hou and Guangxin Yue, "Pilot Aided LS Channel Estimation in MIMO-OFDM Systems" The 8th International Conference on Signal Processing,vol.3, pp.(16-20), 2006.

[10] Reza Abdotee, Tharek Abd.Rehman and Savia Mahdaliza Idrus, "Computational Complexity Reduction for MIMO-OFDM Channel Estimation Algorithm ", elecktika Journal of electrical engineering, vol.9,No.1, pp.( 30-36), 2007.

[11] Bellido, Deseada, Entrambasaguas and Jose T. "MSE evaluation at reception end in MIMOOFDM systems using LS channel estimation", Waveform Diversity and Design Conference, vol.1, pp. (174-177), 4-8 June,2007.

[12] Markus Myllyla, Markku Juntti, Matti Limingoja,Aaron Byman and Joseph,R. Cavallaro "Performance Evaluation of Two LMMSE Detectors in a MIMO-OFDM Hardware Testbed", Conf.on Signals, systems and Computers, Fortieth Asilomar Pacific Grove, pp. (1161-1165), Oct29Nov1 2006.

[13] Ian Griffiths "FPGA Implementation Of MIMO Wireless Communications System", University Of New Castle, Australia, $1^{\text {st }}$ November, 2005.

[14] Bernard Sklar "Digital Communications-Fundamentals and Application" Published by PEARSON Education. Year 2003.

[15] G.Proakis, Masoud Salehi "Communication System Engineering”, Second edition by John. Published by Pearson Education. 2002.

[16] John G. Proakis, Dimitris G. Manolakis, "Digital Signal Processing Principles Algorithms and Applications", $4^{\text {th }}$ Edition, Boston: McGraw Hill.

[17] Himanshu Bhatnagar - "Advanced ASIC Chip Synthesis Using Synopsys ${ }^{\circledR}$ Design Compiler ${ }^{\mathrm{TM}}$ Physical Compiler ${ }^{\mathrm{TM}}$ and PrimeTime ${ }^{\circledR}$ ", Kluwer academic publishers, Second edition - 2002.

[18] David Garrett, Linda Davis, Stephen ten Brink, Bertrand Hochwald and Geoff Knagge, "Silicon Complexity for Maximum Likelihood MIMO Detection Using Spherical Decoding",IEEE journal of Solid State Circuits, vol.39, no.9, pp. (1544-1552), September, 2004.

[19] Yvo L.C. de Jong and Tricia J. Willink, "Iterative Tree Search Detection for MIMO Wireless Systems", IEEE Transactions on Communications, vol. 53, no.6, pp. (930-935), June, 2005.

Veena M.B.: received the B.E.,degree in Electronics \& communication from Mysore university in 1991 and the M.E.,degree in Electronics \& communication from Bangalore university in 2003.Currently pursuing Ph.D from V.T.U., Belgaum, India.

Presently working as a Assistant professor, Dept. of Telecommunication Engineering, Vemana Institute of Technology,Bangalore,India. \& research interest in the field of MIMO wireless communication system, circuits \& systems, Signal processing \& VLSI.

M.N.Shanmukha swamy : received the B.E. \& M.E.,degree in Electronics \& communication from Mysore university. Ph.D from IISC, Bangalore, India. Presently working as a professor, Dept of Electronics \& communication Engineering, SJCE, Mysore, India. \& research interest in the field of wireless communication system, circuits \& systems, VLSI. Adhoc networks \& Image processing. Under his guidance many research students are working. 